Revista de Matemática: Teoría y Aplicaciones 2009 16(1) : 1-15

CIMPA - UCR ISSN: 1409-2433

\title{
EL MÉTODO STATIS COMO ALTERNATIVA PARA DETECTAR "RESPONSE SHIFT" EN ESTUDIOS DE CALIDAD DE VIDA RELACIONADA CON LA SALUD
}

\author{
María Purificación Vicente Galindo* \\ Purificación Galindo Villardón ${ }^{\dagger}$
}

Recibido/Received: 20 Feb 2008 - Versión revisada/Revised version: 24 Nov 2008 - Aceptado/Accepted: 25 Nov 2008

\begin{abstract}
Resumen
Evaluar la Calidad de Vida de los pacientes es difícil porque junto al cambio real (alpha) muchos pacientes que experimentan un cambio en su salud (diagnostico de cáncer, por ejemplo), pueden cambiar sus estándar internos de medida (recalibración), sus valores (repriorización), o incluso reconceptualizar lo que antes entendían por Calidad de Vida (Cambios Beta y Gamma). Este fenómeno es conocido como "Response Shift" y justifica la paradoja descrita en la literatura por la cual pacientes con graves problemas de salud perciben una Calidad de Vida más alta de la estimada por médicos y familiares.

En este trabajo, después de evaluar los problemas existentes a la hora de definir el constructo Calidad de Vida, centramos la atención en las dimensiones del Response Shift. Se discuten las limitaciones del método clásico de Ahmavaara y se proponen soluciones para detectar y evaluar las componentes Beta y Gamma del Response Shift.
\end{abstract}

Palabras clave: Calidad de Vida, Response Shift, cambio Beta, cambio Gamma, método Statis Dual.

\begin{abstract}
After evaluating problems in conceptualisation of Quality of Life outcomes and establishing an operational definition of HRQoL, we focus the attention on the different

\footnotetext{
*Departamento de Estadística, Universidad de Salamanca, Salamanca, España. EMail:purivic@yahoo.com

${ }^{\dagger}$ Departamento de Estadística, Universidad de Salamanca C/ Espejo No 2, 37007 Salamanca, España. E-Mail:E-mail: purivic@yahoo.es
} 
dimensions of Response Shift and the theoretical Models to explaining it. We pay special attention to Schwartz and Sprangers Model.

If Gamma change is present, the construct under assessment is not stable for the patient; so it has no sense to compare scores because if an individual changes conceptualization of QoL over the course of time, then answers to the same items by the same individual may not be as comparable over time as originally thought. So, it is crucial to capture Response Shift before Alpha change is evaluated.

The analytical approaches to assess Response Shift are discussed, paying special attention to the statistical aspects. We found several shortcomings of Ahmavaara's Method for comparing factorial structures and we have developed some alternatives to capture Beta and Gamma components of Response Shift.

Keywords: Health Related Quality of Life (HRQoL), Response Shift, Beta change, Gamma change, Dual Statis method.

\section{Mathematics Subject Classification: $62 \mathrm{H} 25$}

\section{Introducción}

A medida que en las sociedades desarrolladas se ha ido disminuyendo la mortalidad prematura, los índices de cantidad de vida, por ejemplo la esperanza de vida, han ido perdiendo sensibilidad para monitorizar el nivel de salud de la población, y el interés por la cantidad de vida ha dado paso al interés por la Calidad de Vida. Lo mismo sucede en aquellos casos en los que no es posible encontrar un tratamiento efectivo que resuelva realmente el problema (determinados casos de cáncer, por ejemplo). La muerte es segura y por tanto los análisis de supervivencia no son el objetivo primordial. Sin embargo, esta nueva forma de abordar los estudios sociológico epidemiológicos conlleva una serie de nuevos problemas, desde el punto de vista metodológico, que requieren ser abordados y resueltos: ¿Cómo medir la Calidad de Vida?, ¿Con qué instrumentos?, ¿Cómo analizar los datos teniendo en cuenta que el constructo en estudio es eminentemente dinámico y claramente multidimensional?, etc.

Los pocos estudios que existen en España al respecto abordan aspectos parciales, relacionados con evaluaciones concretas de Calidad de Vida en colectivos particulares, o bien con la construcción de instrumentos para evaluar la CVRS, pero no tenemos conocimiento de ningún trabajo que aborde el problema desde el punto de vista metodológico.

Los análisis estadísticos en los trabajos publicados se limitan a la validación de los instrumentos (utilización de los métodos factoriales confirmatorios clásicos para comprobar la dimensionalidad del constructo que se pretende evaluar con el cuestionario, seguidos de análisis de consistencia interna de las estructuras. Una vez evaluadas las características del instrumento de medida para el análisis de los datos la metodología más utilizada (aparte de los análisis básicos de resumen) es el Análisis de la Varianza, bien repitiendo análisis para cada uno de los tiempos evaluados y comparando los resultados, o bien con un análisis de la varianza de medidas repetidas o con MANOVAS. El resto de las publicaciones están centradas en la discusión del problema de los datos faltantes (y/o no respuesta) y en la propuesta de formas de análisis para paliar el sesgo correspondiente. Sin embargo en ninguna publicación se contempla la posibilidad de analizar las estructuras de 
covariación de las respuestas a los items, independientemente de los individuos observados, ni las estructuras de similitud en grupos con morbilidad y Calidad de Vida comparables, para distintos conjuntos de items producidos por cuestionarios diferentes, a pesar de que cualquier política sanitaria requiere el reconocimiento de patrones de comportamiento y percepción y no respuestas particulares. Nuestra aportación metodológica se centra sobre todo en esta dirección, y es absolutamente novedosa.

\section{2 ¿Qué se entiende por Calidad de Vida?}

El interés por la Calidad de Vida ha existido siempre pero la aparición del concepto como tal es reciente. En los años sesenta y setenta los científicos sociales empezaron a mostrar interés por la Calidad de Vida como consecuencia de la percepción de las desigualdades en la distribución de los recursos y el bienestar de la sociedad y la preocupación por el crecimiento de la población en los países subdesarrollados: se inicia el desarrollo de los indicadores sociales como herramienta para evaluar datos vinculados al bienestar social de una población. Estos indicadores tuvieron una evolución que va desde centrar el objetivo en condiciones objetivas de tipo económico y social hasta contemplar elementos subjetivos, hecho éste que provocó la diferenciación entre los indicadores sociales y la Calidad de Vida la cual hace referencia tanto a condiciones objetivas como a componentes subjetivos (Setien (1993) [26]).

En 1974 se incluye el término Calidad de Vida en la revista "Social Indicators Research" de E.E. U.U. y en 1979 se incluyó en el "Sociological Abstracts". En el contexto de la salud, este concepto se convirtió desde 1977, en categoría de búsqueda en el Index Medicus y en palabra clave en la base MEDLINE. Estos hechos contribuyeron a su difusión teórica y metodológica y ya en la década de los 80 se produjo el despegue definitivo de la investigación en torno a este término. Paradójicamente, transcurridos 20 años, aún no existe consenso ni en la definición del constructo ni en la forma más adecuada de medida.

\subsection{Calidad de vida relacionada con la salud}

También en el campo médico, el concepto de Calidad de Vida ha ido sufriendo una evolución constante caracterizada por una continua ampliación. En las antiguas civilizaciones se asociaba con el cuidado de la salud personal, luego se centró en la preocupación por la salud pública, extendiéndose después a los derechos humanos, laborales y civiles, incluyendo a lo largo del tiempo el entorno social, la actividad cotidiana del individuo y su propia salud. Se ha pasado, por lo tanto, desde un concepto relacionado con el entorno y las condiciones de vida hasta la propia percepción y valoración de estas condiciones.

A la mayoría de la gente le resulta familiar la expresión "Calidad de Vida" (abreviadamente CV) y tiene una idea intuitiva de lo que significa. Sin embargo, es muy difícil dar una definición de Calidad de Vida, porque significa diferentes cosas para diferentes personas, para las mismas personas en diferentes momentos e, incluso, tiene diferentes significados dependiendo del área de aplicación.

Al intentar definir el concepto de Calidad de Vida existen dos posturas claramente diferenciadas: la de aquéllos que la entienden como un concepto ideográfico, en el sentido 
de que es el individuo quien debe establecer sus dimensiones, y los que piensan que debe establecerse un criterio general sobre Calidad de Vida para todos los sujetos. Lo que es indiscutible es que el sujeto tiene una percepción subjetiva de sus condiciones que influye en su Calidad de Vida. De acuerdo con el grupo para la Calidad de Vida de la organización mundial de la salud ${ }^{1}$ (WHOQoL), la Calidad de Vida es: "un concepto amplio que incorpora, de forma compleja, la salud física de los individuos (síntomas subjetivos como el dolor, la fatiga, dificultades para caminar, etc.) estado psicológico, nivel de independencia, relaciones sociales (limitaciones en los roles, conexiones sociales, etc.), creencias personales, y sus relaciones con características sobresalientes del ambiente (ahorros, finanzas, transporte, educación, etc.)." En el contexto de ensayos clínicos, Calidad de Vida está relacionada con la evaluación de aquellos aspectos que están afectados por la enfermedad o por su tratamiento.

La definición de Calidad de Vida es problemática. Se han usado numerosas aproximaciones pero ninguna ha sido universalmente aceptada. Consecuentemente, la elección de una definición no es nada simple. Una búsqueda "on line" hecha por Haas (1999) [12] de la frase "Quality of Life" en Medline, Cinahl, Psyc-Info, Eric, y Social Science Abstract, proporcionó una lista de 16.021 artículos publicados entre 1993 y 1998 . De esos artículos, más de 4.000 eran sobre CVRS. Una muestra conveniente de 65 artículos teóricos y prácticos desde 1990 fue examinada por Haas para determinar el uso actual y las diferentes definiciones del concepto de CV en las diferentes disciplinas. El examen de los artículos teóricos publicados en los noventa reveló que la mayoría de los autores definen CV conceptualmente. Salvo en la literatura teórica, no existe una definición explícita de CV. En los trabajos empíricos, los autores no definen $C V$ sino que se limitan a discutir las distintas formas en la que el término es definido y usado por otros. Incluso muchos autores incluían la frase CV en el título del artículo o introducción sin posteriores discusiones o definición. Normalmente la definición implícita viene determinada por el instrumento seleccionado para su medida. La siguiente definición de CV propuesta por Haas [12] es la que nosotros hemos utilizado en este trabajo:

Calidad de Vida es una evaluación subjetiva de bienestar físico, psicológico y social.

Las medidas subjetivas de bienestar no tienen un punto de referencia natural, sino que está determinado por situaciones específicas del individuo (cambios en el estatus de salud del individuo pueden producir procesos cognitivos afectivos y de comportamiento necesarios para acomodar la falta de salud), por las características del individuo (sexo, edad, educación, personalidad, etc.); etc.

Teniendo en cuenta la definición de CV dada por Haas, y que los términos de referencia por los cuales se juzga el bienestar son diferentes para diferentes personas y cambian con el tiempo, en nuestro trabajo Calidad de Vida será entendida como un constructo multidimensional, dinámico, que se altera en respuesta a la enfermedad. Las diferentes dimensiones estarán en relación con el instrumento usado para evaluar la Calidad de Vida.

\footnotetext{
${ }^{1}$ WHOQOL Group, 1995. The world Health Organization Quality of Live Assesment position paper from the World Health Organization. Social Science and Medicine, 41, 1403.
} 


\section{3 "Response shift" en Calidad de Vida relacionada con la salud}

\subsection{Cambios Alpha, Beta y Gamma}

Golembiewski et al. [11] en 1976 conceptualizaron tres tipos de cambios distintos que pueden producirse como resultado de una intervención, a los que llamaron Alpha, Beta y Gamma.

- Cambio Alpha: implica una variación en el nivel de algún estado existencial, considerando un instrumento de medición constantemente calibrado, relacionado con un dominio conceptual constante. Este tipo de cambio es el convencional y es justo el concepto opuesto a la estabilidad cuantitativa, o estabilidad de nivel, descrita por Mortimer et al. [21] en 1982. Sin embargo, Golembiewski et al. aluden expresamente a que el instrumento de medida debe estar constantemente calibrado.

- Cambio Beta: se refiere a la variación en los intervalos de medida de las dimensiones de una realidad estable (recalibración). En estudios de Calidad de Vida es muy frecuente este tipo de recalibración a la que hemos aludido previamente ya que los pacientes pueden hacer una diferente estimación de la realidad, o pueden tener una percepción diferente de la misma debido a procesos de adaptación, por ejemplo.

- Cambio Gamma: implica, además, una redefinición o reconceptualización de las dimensiones de la realidad. Cuando no existe invarianza estructural puede haberse producido un cambio beta o el cambio gamma. Evidentemente una recalibración del instrumento puede evidenciarse cuando el constructo es estable y cambian las unidades del instrumento de medida, pero también puede suceder sencillamente porque el constructo es otro y lleva asociadas medidas diferentes.

Cuando usamos instrumentos para evaluar Calidad de Vida, se presupone siempre que el punto de referencia no se mueve; es decir que la actitud del paciente hacia un constructo concreto permanece estable a lo largo del tiempo que dura el tratamiento o la observación concreta que estemos llevando a cabo. Sin embargo, hay muchas referencias bibliográficas que afirman que, este supuesto es falso. Uno de las más interesantes es el trabajo de Allison et al. [2] publicado en 1997. En este trabajo los autores afirman que cambios en la respuesta a determinadas variables no siempre pueden ser interpretadas en términos de diferencias producidas por el tratamiento, porque las actitudes de los individuos no son constantes, sino que varían con el tiempo y las experiencias vividas pueden ser modificadas por fenómenos psicológicos como la adaptación, las expectativas, el autocontrol, etc.

Muchos autores coinciden en afirmar que lo primero que debe hacerse es descartar un cambio Gamma y una vez descartado tratar de detectar cambios Beta. Sólo cuando estemos seguros de que los dos cambios antes descritos no se han producido tendrá sentido evaluar el verdadero cambio Alpha. En este sentido se manifiesta Golembiewski et al. (1976) [11], Allison et al. (1997) [2], Taris (2000) [27], Bartholomew (2002) [3], entre otros muchos. 


\section{2 "Response Shift"}

Howard \& Daily (1979) [16] introducen el concepto de "Response Shift" en la investigación en educación, para referirse al cambio en los estándares de medida, o del marco de referencia del individuo, desde una medida a otra. Conceptualizan el "Response Shift" como un sesgo que ocurre entre una intervención y otra, que contamina los verdaderos valores del cambio. Este concepto tiene un paralelismo con el concepto de cambio Beta en la tipología de Golembiewski, aunque para estos autores el cambio Beta es un resultado interesante en si, mientras que para Howard \& Dailey es simplemente un sesgo. Estos autores no propusieron ninguna idea similar al cambio Gamma. Presumiblemente, los autores pensaban que la ausencia de "Response Shift" sería análoga al concepto de cambio Alpha de Golembiewski y col.

Schwartz \& Sprangers (1999) [25], introducen el concepto en el campo de la Salud y entienden el "Response Shift" como un cambio en el significado de una auto evaluación que contiene tres componentes: recalibración, repriorización (cambio en valores) y reconceptualización.

Por tanto, para Howard et al. "Response Shift" tenía una sola componente, recalibración (constructo estable, cambios en la escala y/o punto de referencia); para Golembiewski et al. puede tener dos componentes, recalibración (constructo estable, cambios en la escala y/o punto de referencia); y reconceptualización (cambio en el constructo), y para Schwartz y Spranger, tres componentes, recalibración (constructo estable, cambios en la escala y/o punto de referencia); repriorización (cambio en los valores) y reconceptualización (cambio del constructo). Para Schwartz \& Sprengers, "Response Shift" puede ser un resultado de interés en sí mismo, por ejemplo, en pacientes con enfermedades terminales o enfermedades crónicas el resultado de interés no es la curación sino el encontrar la forma de que el individuo tenga la CV más alta posible, dadas sus circunstancias. En estos casos una readaptación de los estándares e incluso una reconceptualización de lo que significa CV es imprescindible y el propiciarlo puede ser un fin, independientemente de si sesga, o no, los resultados en relación a la medición anterior efectuada al individuo. En este mismo sentido se pronuncia Wilson (1999 y 2000) [28, 29]. Evidentemente también en otros casos es un sesgo que puede distorsionar los resultados.

\subsection{Modelo teórico de Schwartz \& Sprangers para explicar "Response Shift"}

Schwartz \& Sprangers (1999) [25] proponen un modelo teórico que explica la presencia o ausencia de "Response Shift" como resultado de un catalizador y la interacción de antecedentes (características estables del individuo como su personalidad, por ejemplo) y de diferentes mecanismos (procesos de ajuste a la situación). Esta interacción lleva al "Response Shift" el cual afecta a la Calidad de Vida percibida por el paciente. El modelo presenta cinco componentes fundamentales: Catalizador (un cambio en el estado de salud, ya sea resultado o no de un tratamiento), Antecedentes (características y predisposiciones del individuo, - personalidad, educación,...-), Mecanismos, (procesos afectivos de comportamiento y de bienestar que utiliza el individuo para adaptar el 
catalizador -reconstruir expectativas, buscar apoyo social...-), Response Shift (un cambio en el significado de la auto evaluación de la CV como resultado de cambios en los estándares internos, en los valores y en la conceptualización de la CVRS) y Calidad de Vida percibida (entendida como un constructo multidimensional que incorpora, al menos tres dominios: físico, social y psicológico).

Para acomodar el catalizador, cada paciente desarrolla procesos de comportamiento concretos; es decir, desarrolla diferentes mecanismos para enfrentar la situación. La literatura es muy rica en relación a este punto; aquí resaltamos las más relevantes: Adaptación, Heyink (1993) [14]; Afrontamiento, Schwartz \& Daltroy (1999) [24]; Respuesta emocional, Haes et al. (1992) [13]; Incertidumbre, Mishel (1988) [20]; Auto-Control, Carver \& Scheier (1982) [5]; Auto-concepto, Foltz (1987) [9]; Expectativas, Daltroy et al. (2000) [7]; Optimismo, Scheier \& Carver (1985) [?]; Comparación con otros (Social Comparison), Gibbons (1999) [10]; Ayuda a los demás (Peer Support), Schwartz \& Sendor (1999) [23]; Experiencias previas, Daltroy (1999) [6].

\section{Métodos para detectar "Response Shift" en datos longi- tudinales}

\subsection{Métodos para detectar Cambio Gamma}

Siguiendo a Golombiewski et al. (1976), la ausencia de cambio Gamma se puede constatar como congruencia de las estructuras factoriales en los diferentes tiempos del estudio. Para estos autores, dos estructuras son iguales cuando tienen el mismo número de factores, y los factores están configurados por las mismas cargas factoriales, pero no es necesario que la magnitud de los factores de carga se mantenga. Estos autores sugirieron que la comparación entre las estructuras factoriales podía hacerse calculando el coeficiente de congruencia dado por Ahmavaara [1] en 1954, el cual consiste, básicamente, en rotar una de las matrices factoriales en el espacio de la otra, para compararlas. La matriz de transformación contiene los cosenos de los ángulos entre los factores de ambos estudios. Ver esquema de la figura 1.

El Método de Ahmavaara presenta las siguientes limitaciones:

- Es un procedimiento para comparar dos estructuras, luego cuando queremos comparar las estructuras en más de dos tiempos no podemos conseguir una visión global del problema.

- Para comparar evolución a lo largo del tiempo hemos de tener los mismos individuos y los mismos ítems (variables).

- Comparar patrones implica calcular muchas correlaciones (producto-momento y/o intraclase) lo cual conlleva, como es bien sabido, a importantes incrementos en el riesgo tipo I; es decir a declarar correlación significativa donde realmente no la hay. 


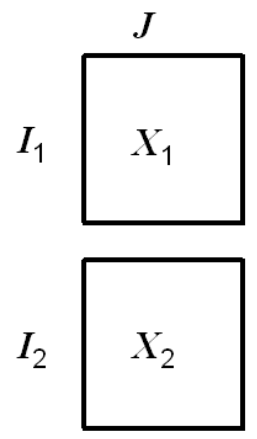

Tablas de

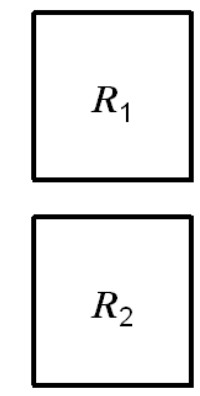

Matrices de
correlación
$R_{t}$

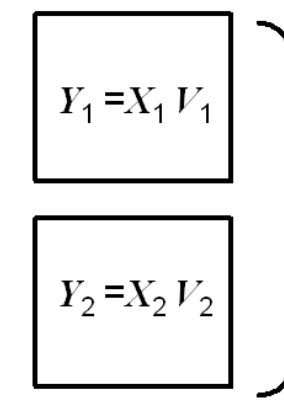
Componentes principales definidas por los primeros vectores propios de $R_{t}$

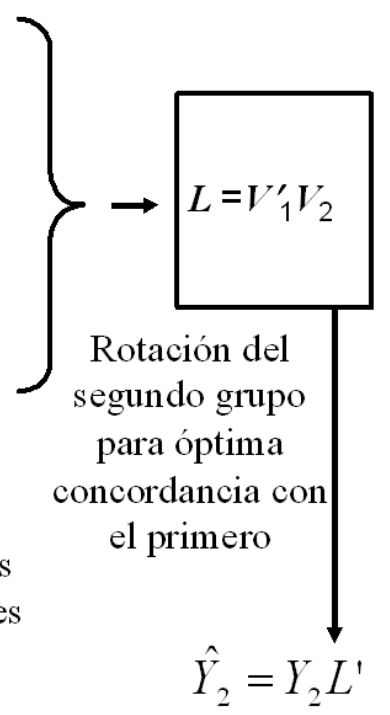

Figura 1: Esquema del desarrollo analítico del Método de Ahmavaara.

\subsection{Statis Dual como herramienta para la detección de "Response Shift"}

\subsubsection{Statis Dual y Calidad de Vida}

Statis Dual, (L'Hermier des Plantes (1976) [19], Lavit (1988) [17]; Lavit et al. (1994) [18]) es un método de análisis para matrices de datos de tres vías que puede ser utilizado para detectar y evaluar "Response Shift" en el sentido de Golombiewski. Veamos.

a) Los datos de Calidad de Vida relacionada con la salud longitudinales, pueden ser entendidos como datos de tres vías: el primer modo se refiere a los pacientes, el segundo a los ítems del cuestionario utilizado para evaluar Calidad de Vida, y el tercero se refiere a las distintas ocasiones en las que la $\mathrm{CV}$ se evalúa. Ver esquema en la figura 2. $X_{t}$ será la matriz de datos en un tiempo concreto $t . X_{t}$ tiene $I$ filas, una para cada paciente, y $J$ columnas, una para cada ítem. $T$ representa el número de ocasiones en las que se ha llevado a cabo el estudio, por ejemplo, en el momento del diagnóstico de un cáncer, después de la intervención quirúrgica, después de dos semanas de quimioterapia, etc.

b) La información para cada tiempo $\left(X_{t}\right)$ puede ser capturada por dos configuraciones diferentes. 
- El producto escalar entre individuos:

$$
W_{t}=X_{t} X_{t}^{\prime}
$$

- La matriz de covarianzas/correlaciones entre ítems.

$$
C_{t}=X_{t}^{\prime} X_{t}
$$

Dependiendo del objetivo del estudio tomaremos como punto de partida en el análisis, una u otra configuración. En nuestro caso, como lo que pretendemos en este apartado es poner de manifiesto que el método puede ser utilizado para detectar Response Shift, trabajaríamos con la configuración $C_{t}$ ya que comparar configuraciones debe ser entendido en este caso como comparación de matrices de covarianzas y/o correlaciones, en las distintas ocasiones.

c) Response Shift puede ser evaluado comparando configuraciones.

Para comparar configuraciones, $C_{t}$ (o $W_{t}$ ) hemos de definir un coeficiente de correlación entre matrices ("el coeficiente de correlación vectorial de Hilbert-Schmidt" $(R V)$ propuesto por Escoufier (1973) [8]) y/o una distancia entre ellas. Para ello necesitamos definir un producto escalar entre pares de matrices (el producto de Hilbert-Schmidt); este producto escalar induce una norma y por tanto una distancia. Si la correlación vectorial entre dos matrices es igual a la unidad, eso significa que ambas matrices son equivalentes en el sentido de que ambas estructuras son congruentes y comparables; es decir no aparecerían discrepancias entre las estructuras factoriales de los dos (o más) tiempos Por tanto, si la correlación vectorial entre las matrices factoriales de dos tiempos (en el sentido de Hilbert Schmidt), es igual a la unidad ambas estructuras serían coincidentes; cuanto más próximo a uno, más similares las estructuras.

El Método Statis Dual permite representar cada matriz de datos de partida correspondiente al estudio en un tiempo (fase del tratamiento, por ejemplo), como un punto en un espacio vectorial de baja dimensión en el cual la proximidad entre puntos se interpreta en términos de similitud entre las estructuras de covarianza de las variables, y por tanto, en términos de congruencia entre las estructuras factoriales. En esta representación, la proximidad entre puntos significa que las matrices tienen la misma estructura de covariación entre los ítems. Ver esquema en la figura 2.

Si las estructuras son comparables se puede calcular una matriz "compromiso" que representa a todas las matrices de partida, correspondientes a las distintas fases del estudio, y sobre ella proyectar los datos originales para poder analizar el comportamiento de los ítems y su evolución a lo largo del tiempo.

d) Statis Dual y Response Shift.

En apartados anteriores hemos descrito el Método de Ahmavaara y hemos puesto de manifiesto que permite únicamente la comparación de parejas de matrices por lo que no es posible tener una visión multidimensional del problema. Además presenta la limitación de que la comparación de las estructuras la establece comparando los 
ejes factoriales por parejas sin explorar si un eje (por ejemplo el primero) tiene baja correlación con el equivalente de la otra matriz pero puede ser absolutamente idéntico a otro cualquiera de la segunda matriz. Evidentemente, para el tema que a nosotros nos ocupa, que es el estudio de la posible reconceptualización, el que la variabilidad de los ejes factoriales haya cambiado no quiere decir que se haya reconceptualizado el constructo latente "Calidad de Vida" ya que sigue teniendo los mismos componentes.

La alternativa que nosotros proponemos basada en el Método Statis nos permite representar geométricamente la estructura de covariación como un punto en un espacio euclídeo y analizar la congruencia global de las estructura "mirando" simplemente la distancia entre los puntos que representan las matrices, y los ángulos que forman al unirlos con el origen de coordenadas del plano euclídeo de máxima inercia, es algo que facilitará mucho el uso de estas técnicas ya que no podemos olvidar que quienes estudian Calidad de Vida no son precisamente estadísticos. Ver esquema en figura 2.

Sometiendo los datos a un análisis Statis Dual y analizando la imagen euclídea de la Inter-estructura, podemos concluir que:

- Si en la imagen euclídea se observa que los ángulos que forman los vectores que unen el origen con los puntos que representan a las matrices están próximos a cero grados, eso significa que las estructuras factoriales de las diferentes matrices son iguales, por tanto hay razones para suponer que no se ha producido una reconceptualización con el paso del tiempo. Dicho de otra manera: si se ha producido un cambio Gamma a lo largo del tratamiento, la representación euclídea de las matrices debe exhibir algún ángulo grande; al menos alguno de los puntos debe estar alejado del resto.

- Si además en la representación euclídea, las normas son iguales, eso quiere decir que tampoco hay razones para pensar que se ha producido una recalibración.

- Si en la representación euclídea se pone de manifiesto que todas las matrices tienen la misma estructura factorial, se puede calcular una matriz compromiso (media) que nos dé una visión global de las dimensiones latentes del constructo Calidad de Vida, a lo largo de todo el tratamiento.

- Si la representación euclídea pone de manifiesto que alguna de las estructuras es diferente, y por tanto, que cabe suponer que la concepción del constructo ha cambiado, no podremos comparar los resultados antes y después del tratamiento para cuantificar el cambio Alpha ya que está enmascarado por el cambio Gamma.

- Lo mismo sucederá si se pone de manifiesto que hay razones para pensar que se ha producido una recalibración ya que en ese caso tampoco será interpretable el cambio entre el pre y el post tratamiento ya que estará sesgado por el cambio Beta.

- Sobre la matriz compromiso se pueden proyectar los ítems de las matrices originales para analizar sus trayectorias a lo largo del tiempo. Esto nos puede ayudar a detectar los ítems implicados en la reconceptualización y en la recalibración. 


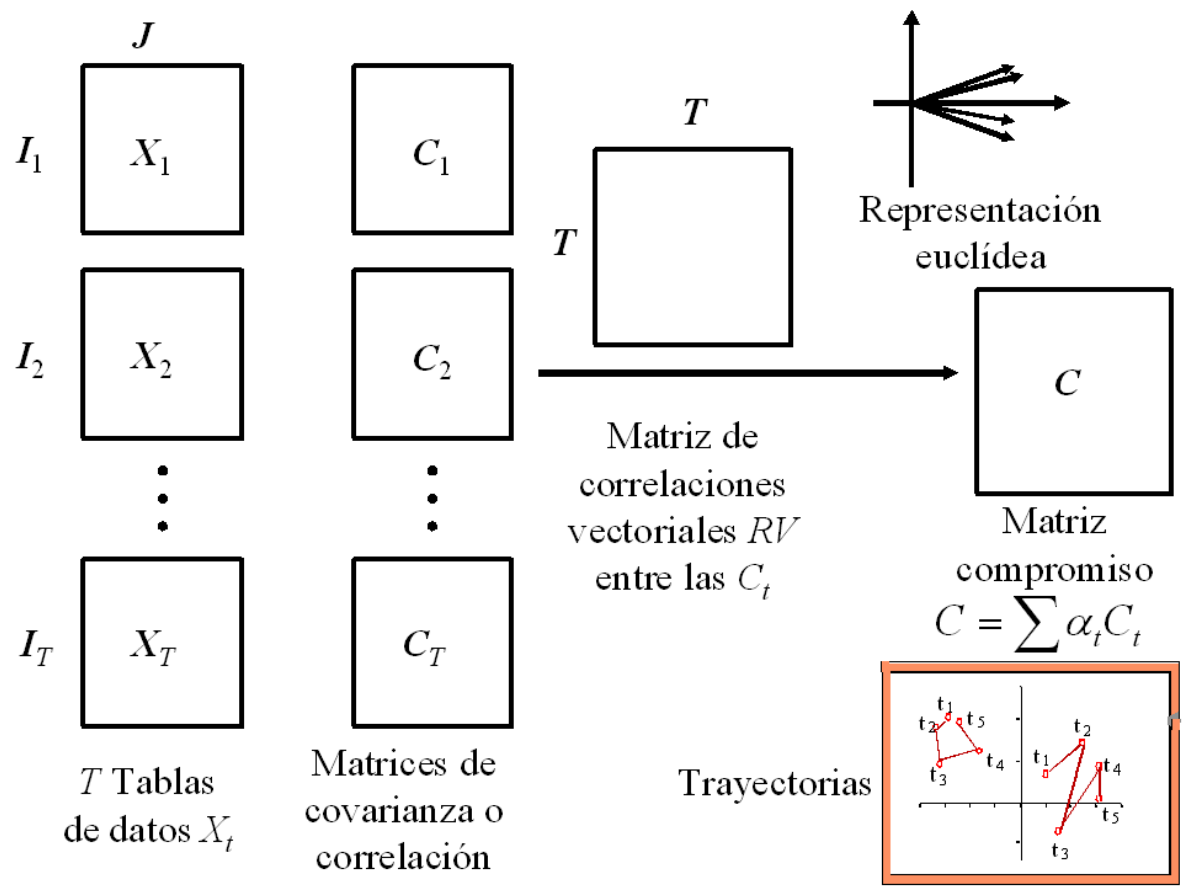

Figura 2: Esquema con los pasos del proceso algebraico en el que está basado el Statis Dual.

\section{Métodos para detectar cambio Beta}

Golembiewski et al. [11] no propusieron una metodología específica para evaluar cambio Beta. Sin embargo, el cambio Alpha no puede ser evaluado hasta tener un constructo constante y un instrumento de medida con escala y origen fijo.

\subsection{El "then-test"}

El método más conocido para evaluar recalibración es el llamado "then-test" el cual fue descrito por Howard et al. (1979) [15]. El "Then-test" se refiere a medidas hechas en el momento del "Post-test" pero referidas al momento del "Pre-test"; es decir se le pide al individuo que haga en el momento presente una evaluación de cómo se sentía en el momento del "Pre-test". La idea en la que se basa es la siguiente: si se ha producido algún tipo de recalibración en la escala de medida entre el "Pre-test" y el "Post-test" ambos valores no serían comparables pero al ser evaluadas las dos experiencias en el momento del "Post-test", la recalibración afectará por igual a ambas evaluaciones. Así pretenden asegurarse de que los estándares de medida para las dos evaluaciones son los mismos y evitar que el cambio Beta sesgue el verdadero cambio Alpha. Si aparecen diferencias entre el "Pre-test" y el "Then-test" se asume que se ha producido un cambio Beta. La diferencia 
entre los valores al realizar el "then-test" y el post-test se acepta como verdadero cambio Alpha. Desde el punto de vista estadístico la comparación es trivial; una t de Student para datos apareados resuelve el problema de la comparación, tanto del Pre-Post-test como del Then-test - Post-test. En realidad lo importante es que los valores que se comparan no estén sesgados.

\subsection{Cambio Beta en presencia de cambio Alpha}

Ya hemos señalado más arriba que puede detectarse un cambio Beta que puede ser debido a una reconceptualización del concepto y no a una simple recalibración de los estándares de medida. Por eso nosotros proponemos:

- Antes de interpretar las diferencias entre el "Pre-test" y el "Then-test", comparar las estructuras factoriales, correspondientes a ambos tiempos para descartar la presencia de cambio Gamma.

- Una vez descartado el cambio Gamma, evaluar el cambio Beta a través de las diferencias entre el Pre- y el Then-test.

- Una vez descartado el cambio Gamma, evaluar el cambio Alpha a través de las diferencias entre el Then y el Post. Si el cambio Gamma está asociado a una reconceptualización del constructo, no tiene sentido comparar valores porque si ha cambiado el concepto, respuestas a los mismos ítems pueden no ser comparables.

\subsection{Comparación de estructuras factoriales y cambio Beta}

La comparación de estructuras factoriales también proporciona información sobre la posible presencia de un cambio Beta, aunque esto no haya sido aplicado prácticamente en los trabajos relacionados con la salud. Una vez que hemos comprobado que las estructuras factoriales presentan el mismo patrón; es decir tienen el mismo número de ejes y presentan los mismos ítems con altos factores de carga en cada eje, podemos analizar la presencia de un cambio Beta analizando la magnitud de los factores de carga y /o las varianzas y covarianzas de las variables latentes y de las variables observables. (Schmitt (1982) [22]; Taris (2000) [27]).

Ya señalamos al hablar del estudio de la Inter-estructura en el método Statis Dual, que si la imagen euclídea de la Inter-estructura ponía de manifiesto que la norma de alguna de las matrices era más pequeña, o más grande que el resto, eso sugería la presencia de un cambio Beta (recalibración de los estándares de medida).

\section{Conclusiones}

El "Response Shift" es un fenómeno clave en la evaluación de la Calidad de Vida relacionada con la Salud, ya que su presencia invalida la medida del cambio debido a un tratamiento; sin embargo, la mayor parte de los trabajos publicados que recogen estudios 
empíricos ni siquiera se plantean su existencia. El "Response Shift" tiene diferentes dimensiones según los diferentes autores y los diferentes campos en los que se ha descrito: para unos traduce un cambio en los estándares de medida, otros añaden a la recalibración del instrumento la reconceptualización del constructo latente, y para otros puede tener una tercera dimensión, relacionada con un cambio en los valores. Los trabajos más actuales de Calidad de Vida relacionada con la Salud asumen las tres dimensiones.

Golembiewski atribuye un cambio Gamma (reconceptualización) a la falta de congruencia entre estructuras factoriales, detectada con el procedimiento de Ahmavaara, pero esa afirmación puede no ser cierta ya que el hecho de que las componentes del constructo latente no coincidan exactamente en el orden de absorción de inercia, no implica que el constructo tengan dimensiones latentes diferentes.

En este trabajo se ha propuesto una alternativa a la propuesta de Golembiewski basada en el Statis Dual, que nos permite descartar la presencia de "Response Shift", o describir sus componentes, a partir de la correlación vectorial entre matrices y de la distancia de Hilbert Schmidt.

\section{Referencias}

[1] Ahmavaara, Y. (1954) "Transformation analysis of factorial data", Annals of the Academy of Science Fennicae, Series B 881(2): 54-59.

[2] Allison, P.J.; Locker, D.; Feine, J.S. (1997) "Quality of life: A Dynamic Construct", Soc. Sci. Med. 45(2): 221-230.

[3] Bartholomew, S. (2002) Implicit Theories and Beta Change in Longitudinal Evaluations of Training Effectiveness: An Investigation Using Item Response Theory. $\mathrm{PhD}$ Disertation. Virginia Polytechnic Institute and State University.

[4] Carr, A.J.; Gibson, B.; Robinson, P.G. (2001) "Is Quality of Life determined by expectations or experience?", British Medical Journal 322(19): 1240-1243.

[5] Carver, C.S.; Scheier, M.F. (1982) "Control Theory: a useful conceptual framework for personality-social, clinical and health psychology", Psychological Bulletin 92(1): $11-135$.

[6] Daltroy, L.H.; Larson, M.G.; Eaton, H.M.; Phillips, C.B.; Liang, M.H. (1999) "Discrepancies between self reported and observed function in the elderly: the influence of response shift and other factors", Social Science Es Medicine 48: 1549-1561.

[7] Daltroy, L.H.; Larson, M.G.; Eaton, H.M.; Phillips, C.B.; Liang, M.H. (2000) "Discrepancies between self reported and observed function in the elderly: the influence of response shift and other factors", (In Adaptation to Changing Health. Response Shift in Quality-of-Life Research. Ed. American Psycological Association: 189-200).

[8] Escoufier, Y. (1973) "Le traitement des variables vectorielles", Biometrics 29: 750760 
[9] Foltz, A.T. (1987) "The influence of canceron self-copncept and life quality", Seminars in Oncology Nursing 3(4): 303-312.

[10] Gibbons, F.X. (1999) "Social Comparison as a mediator of response Shift", Social Science \& Medicine 48: 1517-1530.

[11] Golembiewski, R.T.; Billingsley, K.; Yeager, S. (1976) "Measuring Change and Persistence in Human affairs: types of changes generated by OD desings", Journal of Applied Behavioural Science 12: 133-157.

[12] Haas, B. K. (1999) "A Multidisciplinary Concept Analysis of Quality of Life", Western Journal of Nursing Research 21(6): 728-743.

[13] Haes J.C.; de Duiter, J.H.; Tempelaar, R.; Pennink, B.J. (1992) "The distinction between affect and cognition in the quality of life of cancer patients-sensitivity and stability", Quality of Life Reseach 1(5): 315-322.

[14] Heyink, J. (1993) "Adaptation and well-being", Psychological Reports 73: 13311342.

[15] Howard, G.S.; Ralph, K.M.; Gulanick, N.A.; Maxwell, S.E.; Nance, D.; Gerber. S.K. (1979) "Internal invalidity in pretest-postest self reporting evaluations and a reevaluation of retrospective present", Applied Psychological Measurement 3: 1-23.

[16] Howard, G.S.,; Dailey, P.R. (1979) "Response-shift bias: A source of contamination of self- report measures", Journal of Applied Psychology 64: 144-150.

[17] Lavit, C.(1988) Analyse Conjointe de Tableaux Quantitatifs. Masson, Paris.

[18] Lavit, C.; Escoufier, Y.; Sabatier, R.; Traissac, P. (1994) "The ACT (STATIS method)", Computational Statistics and Data Analysis 18: 97-119.

[19] L'Hermier des Plantes, H. (1976) Structuration des Tableaux à Trois Indices de la Statistique: Théorie et Application d'une Méthode d'Analyse Conjointe. Thèse de Doctorat de 3ème Cycle, Université des Sciences et Techniques du Languedoc.

[20] Mishel, M.H. (1988) "Uncertainty in ilness", Journal of Nursing Scholarship 20(4): $225-232$.

[21] Mortimer, J.D.; Finch, M.D.; Kumka, D. (1982) "Persistence and change in development: The multidimensional self-concept". in: P.B. Baltes \& O.G. Brim (Eds.) Life-Span Development and Behavior 4, Academic Press, New York: 263-309.

[22] Schmitt, N. (1982) "The use of anriables of covariance structures to assess Beta and Gamma changes", Multivariate Behavioral Research 17: 343-358.

[23] Schwartz, C.E.; Sendor, R.M. (1999) "Helping others helps oneself: respose shift effects in peer support", Social Medecine 48: 1563-1575. 
[24] Schwartz, C.E.; Daltroy, L.H. (1999) "Learning from unreliability: the importance of inconsistency in coping dynamics", Social Science; Medicine 48: 619-631.

[25] Schwartz, C.E.; Sprangers, M.A.G. (1999) "Methodological approaches for assessing respose shift in longitudinal quality of life research", Social Medecine 48: 1531-1548.

[26] Setien, M.L. (1993) Indicadores Sociales de Calidad de Vida. CIS, Madrid.

[27] Taris, T. (2000) A Primer in Longitudinal Data Analisis. Sage, London.

[28] Wilson, I.B. (1999) "Clinical Understanding and Clinical Implications of Response Shift", Social Science; Medicine 48: 1577-1558.

[29] Wilson, I.B. (2000) "Clinical Understanding and Clinical Implications of Response Shift". (In Adaptation to Changing Health. Response Shift in Quality-of-Life Research, Ed. American Psycological Association.) 